\title{
Selenium and Vitamin E for Prostate Cancer - Justifications for the SELECT Study
}

\author{
Venkataraghavan Ramamoorthy ${ }^{1}$, Muni Rubens ${ }^{2 *}$, Anshul Saxena ${ }^{2}$, Nancy \\ Shehadeh $^{3}$
}

\begin{abstract}
There are several studies that relate oxidative damage as possible mechanism for many cancers. Many studies have also shown that anti-oxidants like selenium and vitamin $\mathrm{E}$ decrease the risk for prostate cancer. The main objective of the Selenium and Vitamin E Cancer Prevention Trial (SELECT) study was to look for the benefits of selenium and vitamin $E$ supplementation on prostate cancer. The study had a large sample size, stringent experimental conditions, very long duration, standardized laboratories for biochemical analyses and other factors that contribute to high external validity. The SELECT study failed to show any significant risk reduction for prostate cancers ascribable to selenium and vitamin $E$ supplementations. Because of these conflicting results, many researchers argue about the methods used, supplementations administered (selenium and vitamin $\mathrm{E}$ ) and indicators used for assessing levels of supplementations. We reviewed many epidemiological studies, clinical trials, and pre-clinical studies. With corroborative evidences we justify that SELECT study has a sound methodology and rationale. In lieu of the contrary results of the select study, researchers should focus on the probable mechanisms for these contrary findings and continue their search for newer and effective agents for prevention of prostate cancer.
\end{abstract}

Keywords: Selenium - vitamin E - prostate cancer - SELECT study - oxidative stress - anti-oxidant

Asian Pac J Cancer Prev, 16 (7), 2619-2627

\section{Introduction}

Globally, prostate cancer is the fifth most common cancer in terms of incidence and the fifth most common cause of cancer deaths (WHO, 2008). In the United States, prostate cancer is the most common cancer in men with an estimated lifetime incidence of $16.6 \%$ and $18.1 \%$ for Whites and African-Americans respectively (Brawley, 2012; CDC , 2014). Though incidence rates have started to decline in United States, there were 186,320 new cases and 28,600 deaths in 2008 (Brawley, 2012). A number of studies have shown a variety of predisposing factors like dietary fat, androgen and environmental exposures, with oxidative injury and lipid peroxidation induced by these agents as possible mechanisms underlying these associations (Afanas'ev, 2011). There are many studies that relate oxidative stress to growth and progression of prostate cancer and anti-oxidants like selenium and vitamin $\mathrm{E}$ have shown to decrease risks for prostate cancer (Vlajinac et al., 1997; Heinonen et al., 1998; Wright et al., 2007; Thompson Jr et al., 2013; Yang et al., 2014). The main objective of the Selenium and Vitamin E Cancer Prevention Trial (SELECT) study was to reaffirm this hypothesis with extremely large sample size, stringent experimental conditions, longer duration of study, standardized laboratories for biochemical analyses and other factors that contribute to high external validity. However, the SELECT study did not show any significant association between selenium and vitamin $\mathrm{E}$ supplementation and risk for prostate cancer (Heinonen et al., 1998). On the contrary, a recent analysis of SELECT study published in 2014 showed that selenium supplementation increased the risk for prostate cancer in participants with high baseline selenium levels and vitamin E supplementation increased the risk in participants with low baseline selenium levels (Kristal et al., 2014). Because of these results, many researchers argue about the methods used, supplementations administered (selenium and vitamin E) and indicators used for assessing levels of supplementations. Hence, the main aim of this paper is to justify the SELECT study, by reviewing in detail the methodology as well as the rationale for use of selenium and vitamin $\mathrm{E}$ for prostate cancer.

\section{Epidemiological Disparities and Rationales for Larger Studies on Prostate Cancers}

There are significant differences across various countries with respect to susceptibility to prostate cancers (Miller, 2012). In majority of the Asian countries prostate 
cancer incidences are very low and are not considered a major health problem (Miller, 2012; Xia et al., 2012). In stark contrast to these countries, prostate cancer has been one of the leading causes of cancer deaths in majority of the western countries (Miller, 2012; Xia et al., 2012). Autopsy studies done in several countries have confirmed that $42-80 \%$ of the male population have small foci of dormant prostate cancer without any clinical symptoms by eighty years of age (Venkateswaran and Klotz, 2010). In countries with higher incidence of prostate cancer these micro-foci appear to be more bulky with greater degree of malignant transformations (Sakr et al., 1998). Furthermore, in countries that have higher incidence of prostate cancer, these micro-foci are found in greater numbers and in multiple sites within the prostate gland (Sakr et al., 1998). There are many studies that investigated the status of these micro-foci in populations that have migrated from low incidence countries to high incidence countries (Muir et al., 1991; Shimizu et al., 1991). It is evident from these studies that migrant population form low incidence countries tend to develop more bulky and invasive lesions that tend to approach the ones found in high incidence countries, within a mean calculated stay of 5-6 years in high incidence countries (Sakr et al., 1998). Many studies have attributed these findings to changing dietary and lifestyles factors (Gupta-Elera et al., 2012). Thus, there is a need for larger studies to better understand the role of dietary and lifestyle factors for prostate cancer.

\section{Role of Oxidative Stress in Malignant Transformation Process}

Reactive oxygen species (ROS) alter the rates of several biochemical processes within the cell predisposing towards malignant transformation. ROS are formed endogenously in non-deleterious amounts during normal cellular respiratory processes in the mitochondria. They are produced in large amounts by toxic injuries to the cell due to alcohol consumption, smoking, carcinogens, ionizing radiations, stress and other environmental factors. ROS damage cellular lipid membranes, nuclear DNA, mitochondrial DNA, lipid membranes of intracellular organelles and several other intracellular and extracellular components of the cell (Gupta-Elera et al., 2012).

Several animal studies have shown that fat intake and androgens increase the levels of biological oxidative stress, specifically in prostatic tissues (Meyer et al., 2005; Vykhovanets et al., 2011; Ide et al., 2012). In an observational study done among 650 healthy men aged 50 years and above, increased dietary fat intake was associated with decreased levels of glutathiones-transferase activity in prostatic tissues, indicating increased free radical mediated lipid peroxidation injuries. The byproducts of these peroxidation injuries were also found to aggravate the DNA damage (Meyer et al., 2005; Vykhovanets et al., 2011; Ide et al., 2012). In a study done in mice, it was also found that increased dietary fat and decreased antioxidant levels favored the growth of artificially implanted cancerous prostate tissue in apparently healthy mice. These cancerous tissues were also successful in evading the host immune defense mechanisms in several of these experimental animals (Vykhovanets et al., 2011).

Ripple et al. (1999) through in-vitro studies demonstrated that androgens increase the levels of oxidative stress in human LNCaP prostate cancer cell lines (in vitro human prostate adenocarcinoma cells). Androgen mediated increased oxidative stress could be responsible for the differences found in the prostate specific oxidative damage levels between different races. African Americans have shown greater functional levels of genes that regulate the production, functions and metabolism of androgens than Caucasian males (Kheirandish and Chinegwundoh, 2011). African Americans have also shown greater levels of oxidative damages in their prostatic tissues than the Caucasian white population as shown by several cancer survey results (Kheirandish and Chinegwundoh, 2011). Hence, oxidative stress could be the common underlying mechanism for both dietary fat and androgen mediated increased incidence of prostate cancers. Secondary analysis done on data collected from Alpha-Tocopherol, Beta-Carotene Cancer Prevention (ATBC) study showed that vitamin $\mathrm{E}$ supplementation reduced the incidence of prostate cancers by 32 percent (Heinonen et al., 1998). Similarly, secondary analysis of Nutritional Prevention of Cancer (NPC) trails showed that selenium supplementations decreased the incidence of prostate cancers by 51 percent (Duffield-Lillico et al., 2003). These studies also support the role of oxidative stress as a possible mechanism for prostate cancer.

Figure 1 shows the antioxidant mechanism of selenium and vitamin $\mathrm{E}$.

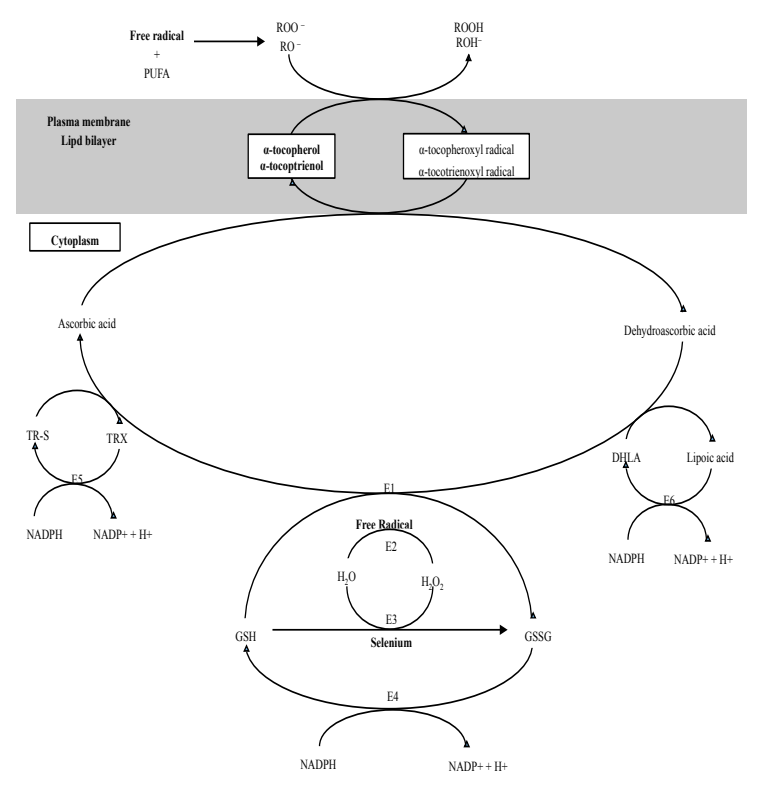

Figure 1. Mechanism of Antioxidant Action of Selenium and Vitamin E. PUFA-Polyunsaturated fatty acid; ROO-Organic peroxyl radical; ROOH-Organic peroxide; TR-S-Reduced thioredoxin (active); TRX-Oxidized thioredoxin; GSH-Reduced glutathione; GSSG-Oxidized glutathione: DHLA-Dihydro lipoic acid; E1-Glutathione-dependent dehydroascorbate reductase; E2-Superoxide dismutase; E3-Glutathione peroxidase; E4-Glutathione reductase; E5Thioredoxin reductase; E6-Lipoamide dehydrogenase 


\section{SELECT Study}

The SELECT study is a phase III double blind placebo controlled clinical trial that investigated the associations between selenium, vitamin $\mathrm{E}$ and prostate cancer. A total of 35,533 healthy men were selected for the study. The initial selection criteria included a digital rectal examination not suspicious for prostate cancer and serum prostate specific antigen (PSA) levels $\leq 4 \mathrm{ng} / \mathrm{ml}$. Age related eligibility criteria were 55 years and above for Caucasian men and 50 years and above for African American men (Klein et al., 2000). This difference in age related eligibility was to account for the greater age related risk among African American men (Powell et al., 1999). African American men have always had higher incidence and mortality due to prostate cancer compared to Caucasian men irrespective of their lifestyles and ethnic differences (Brawley, 2012).

Randomized allocation of study subjects to each of the four arms of the study (Selenium, Vitamin E, Selenium and Vitamin E, and Placebo) assured minimum selection bias. The study was planned for 12 years duration. The initial 5 years involved recruitment of study subjects all across United States, Canada and Puerto Rico. There were a total of 427 sites operating in the two countries. The remaining 7 years were used for interventional aspects of the study (Klein et al., 2000).

Subjects with history of bleeding disorders or on anticoagulant therapy were excluded from the study (Klein et al., 2000). Vitamin E is a powerful antithrombotic agent because it inhibits platelet aggregation (Kim et al., 2011). Studies have shown that vitamin E produces massive conformational changes in glycoprotein IIb/IIIa (Kim et al., 2011). Vitamin E also inhibited thrombin-induced phosphatidyl serine externalization required for platelet aggregation (Kim et al., 2011). Vitamin E produces marked increase in aminophospholipid translocase enzyme activity thereby inhibiting both internal and external coagulation cascades. This justifies the exclusion of participants with bleeding disorders and anti-coagulant therapy from the study.

Another inclusion criteria i.e., serum PSA $<4$ ng/ml needs additional justification (Klein et al., 2000). PSA as a marker for prostate cancer has been very useful in early identification and prompt treatment of prostate cancer. In a randomized study of 182,000 European men, the use of PSA as a screening tool significantly decreased prostate cancer related mortality rate by $20 \%$ when compared to the control group without any intervention (Schröder et al., 2009). In a study among 1,653 men aged 50 years and older, it was found that including serum PSA levels with rectal examination and ultrasonography significantly increased the chances of detecting prostate cancer (Catalona et al., 1991). In the same study, when these three measurements were individually compared for error rates, it was observed that serum PSA levels had the lowest error rate (Catalona et al., 1991). Irrespective of the advantages of PSA, prostate cancer has always been disreputed to be over diagnosed due to extreme sensitivity of this cancer screening tool (Catalona et al., 1991). In a study done by Roemeling et al. (2007), baseline PSA screenings 'over predicted' prostate cancer by as much as $30 \%$ and successive screening after 4 years 'over predicted' by as much as 44 percent. Another study by Steyerberg et al. (Steyerberg et al., 2007) also showed that $49 \%$ of prostate cancers screened by PSA progressed to indolent or harmless cancers. The Prostate Cancer Prevention Trial (PCPT) study, which commenced in 1993 initially a set a cutoff of $\leq 4 \mathrm{ng} / \mathrm{ml}$ as an inclusion criteria for the study because PSA levels $\leq 4 \mathrm{ng} / \mathrm{ml}$ were considered to be extremely low risk for prostate cancer (Thompson Jr et al., 2013). It is therefore justified that even in the SELECT study participants with $\leq 4 \mathrm{ng} / \mathrm{ml}$ of PSA were included in the study.

Selenium was supplemented in doses of $200 \mu \mathrm{g} /$ day as L-selenomethionine and vitamin $\mathrm{E}$ was supplemented in doses of $400 \mathrm{IU} /$ day of dl- $\alpha$ tocopheryl acetate (Klein et al., 2000). Baseline visits were accompanied with blood draws and toenail samples. Participants were followed every 6 months with clinical examinations and relevant questionnaires for assessments of smoking, alcohol consumption and dietary habits. Participants who developed prostate cancer during the course of the study were also requested to undergo biopsies which were confirmed through Central Pathology Review Diagnostic Criteria. Adverse events associated with selenium and vitamin E supplementations were also recorded according to the guidelines established by National Cancer Institute (Klein et al., 2000).

The primary endpoint of the trial was diagnosis of prostate cancer based on Clinical Diagnostic Manual Criteria which included either a positive bone scan and PSA $>50 \mathrm{ng} / \mathrm{ml}$ or a positive histological analysis of biopsied prostate specimen. The secondary endpoints were serum macro- and micronutrient changes with advancing stages of the disease, prostate cancer free survival rates, incidence of other cancers, cardiovascular events, and quality of life measures (Klein et al., 2000).

\section{Rationale for Inclusion of Selenium and Choice of Supplementation in SELECT Study}

Selenium is a component of the glutathione peroxidase enzyme system and protects the body from the oxidative damages of the free radical products of normal respiratory pathway (Swanson et al., 1991). Selenium catalyzes the reduction of oxidative metabolites like lipid peroxides and superoxides that are byproducts of the mitochondrial respiratory pathways. It accomplishes this function by catalyzing 4 types of glutathione peroxidase enzymes which include cellular (GSHPx-1), gastrointestinal (GSHPx-2), plasma (GSHPx-3) and sperm nuclei (snGPx) (Swanson et al., 1991). A number of animal studies have demonstrated that selenium can activate various tumor suppressor genes like p53, VHL, APC and CD95 (Wu et al., 2011). In an in vitro study done on MRC-5 fibroblasts cell line, it was found that selenium was responsible for induction of apoptosis and senescence through activating ataxia telangiectasia mutated (ATM) dependent senescence response factors (Wu et al., 2011). In the same study it was also found that selenium arrests $\mathrm{S}$ and $\mathrm{G} 2 / \mathrm{M}$ cell 
cycle phases thereby preventing cancerous growth (Wu et al., 2011). In another study done on diethylnitrosoamine (DEN) induced Mouse Hepatocellular Carcinoma Cell lineages (MHCCL), administration of selenium produced beneficial effects by increasing the productions of antitumorigenic interleukin, TNF- $\alpha$ (Liu et al., 2012). In a study done on osteosarcoma cell lines, it was observed that administration of selenium in the form of methlyselenic acid (MSeA) was responsible for necrotic death of cells with mutations in Werner syndrome proteins (WSP). WSP act as a caretaker of the genome and mutations result in advanced aging and increased risk for many cancers (Cheng et al., 2012). Selenium is also involved in favorably modulating several epigenetic responses which include methylation of DNA, modification of histones and micro RNAs which collectively decrease the chances of the cell becoming malignant (Ho et al., 2011). Such protective effects of selenium have also been confirmed in normal cells. In an in vitro study done on normal lung fibroblasts and normal colon fibroblasts cell lineages, it was found that selenium administration at hemi-lethal doses induced cellular senescence through the expression of senescence associated beta-galactosidase and 5-bromo2-deoxy uridine incorporation facilitation ( $\mathrm{Wu}$ et al., 2010). Senescence is one of the early protective factors against tumorigenesis. Selenomethionine supplementation has also demonstrated a dose dependent growth inhibition of several human cancer cell lines like MCF-7/S breast carcinoma, DU-145 prostate cancer cells and UACC-375 melanoma (Redman et al., 1998). A number of mechanisms have been hypothesized for the anti-tumorigenic effects of selenium, of which potent antioxidant effects, inhibition of cellular proliferations, enhancement of immune functions and protective effects on DNA damage have gathered much scientific validation (Redman et al., 1998). All these supportive evidences unquestionably justify selenium as an ideal candidate to be tested for prevention of prostate cancer in SELECT study.

SELECT study's rationale for supplementation of selenium in the form of selenomethionine (organic form) is also well justified. A number of studies suggest that organic selenium supplementations have better absorption and tissue incorporation rates than synthetic forms (Levander, 1997). In a review published by Dodig and Cepelak (2004), organic forms of selenium like selenomethionine and high selenium yeast have been justified to be superior to inorganic forms like selenate and selenite due to better absorption, retention and assimilation profiles. Hence, failure of SELECT study to demonstrate any significant reduction in prostate cancer mortality and morbidity cannot be due to the choice of organic selenium. A Brazilian study done in 2011 strongly argues that food sources rich in selenium are always better than any supplemental form (Lemire et al., 2012). Though the superiority of selenium rich foods is scientifically established, SELECT study has managed to follow an intermediate step of supplementing organic selenium because of feasibility and ease of access. Supplementation of selenium rich food would have limited the feasible of the study.

\section{Justification for Selenium Sufficiency Indicators}

In a review published by Levander (1997), plasma selenium levels and whole blood selenium levels were found to be better than serum selenium levels for measuring the adequacy of body selenium status. Serum selenium levels fairly predict selenium adequacy and become comparable to plasma or whole blood selenium after several adjustments (Levander, 1997). In spite of these shortcomings, SELECT study chose serum selenium levels as the marker of selenium status. This could be justified because of certain observations and associations found in other studies. Allen et al. (1999) in his review has observed that in majority of selenium supplementation studies, serum selenium levels fluctuate better than plasma or whole blood selenium, specifically when organic forms of selenium are supplemented or withdrawn. Selenomethionine supplemented in the SELECT study is an organic form of selenium. Fluctuations in serum selenium levels would increase the internal validity of the study because it would facilitate accurate estimation of circulating levels of administered supplement. Fluctuating levels also help measuring participant compliance through intermittent bio-adherence tests (Allan et al., 1999). Whole blood and plasma selenium levels remain stable for a long time irrespective of changes in supplementation dosages or forms. Several selenium depletion studies have shown that whole blood and plasma selenium concentration were not altered for weeks after extremely low selenium diets (Brown and Arthur, 2001).

Many other markers of selenium sufficiency have been recommended by different researchers. Erythrocyte and platelet selenium levels fluctuate more slowly than serum selenium levels and are useful marker for ultra-long effects of selenium supplementation (Neve, 1991). Decrease in erythrocyte and platelet selenium is associated with conditions like cancer cachexia, insufficient oral intake, short bowel syndrome and inadequate parental nutrition (Neve, 1991). Factors associated with prognosis and severity of the disease could be estimated through these markers. However, the primary endpoint of the SELECT study was incidence of prostate cancer confirmed by routine clinical care. Prognosis and severity of the disease were secondary endpoints.

Several studies recommend glutathione peroxidases 1 and 3 in platelets and plasma as better predictors of cellular uptakes and utilization of selenium. However, in a Chinese study done by Xia et al. (1989), it was observed that glutathione peroxidase activity is affected by a number of other factors like exposure to toxic elements, pro-oxidants, carcinogens, ionizing radiations, iron deficiency and strenuous physical activity which are not associated with selenium adequacy (Xia et al., 1989). It is thus justified that glutathione peroxidase level would not be appropriate in the SELECT study.

Additionally, toe nail samples were collected to look for toxic symptoms of hyper-supplementation in SELECT study. Toe nail selenium levels have been used in several large scale studies before SELECT study as a direct marker of selenium adequacy (Yoshizawa et al., 1998). For 
example, in a randomized trial involving 51,529 patients from Boston, toenail selenium levels were used for associations between selenium sufficiency and advanced prostate cancer (Yoshizawa et al., 1998). The SELECT trial could have used toenail levels for the actual study rather than using it as an adjunct marker. However, there are other studies that show that toenails samples would be contaminated by cutters used to produce clippings. Furthermore, selenium levels in nail samples would be leached away by soaps and detergents and solvents used for hygienic purposes (He, 2011).

\section{Rationale for Inclusion of Vitamin $\mathrm{E}$ in SELECT Study}

Vitamin E is a potent lipid soluble antioxidant and primarily functions by dissolving in the cellular lipid bilayer. It functions by scavenging free radicals and thus preventing lipid peroxidation of biological membranes (Laranjinha et al., 1995). It actively reduces highly toxic free radicles, like hydroxyl ions, superoxide ions, lipid peroxyl groups, hydroperoxyl groups, and nitrates. It protects the biological membranes from leaking due to gaps produced by these toxic agents (Burton, 1994). A number of studies have demonstrated the cancer protective effects of Vitamin E. In a study done in androgen independent human prostate PC-3 tumor cell lines, administration of both $\alpha$-and $\gamma$-tocopherols were associated with marked decrease in Cyclin D1 and Cyclin E levels. Vitamin E supplementation also resulted in upregulation and increased activity of trans-glutaminase-2 (TG-2) (Torricelli et al., 2013). Decrease in Cyclin D1 and $\mathrm{E}$ and increased TG-2 indicate decreased cellular proliferation, arrested cell cycle progression, stabilization of cell cycle in the metaphase and thereby lesser chances of uncontrolled proliferations (Torricelli et al., 2013). Vitamin E thus facilitates differentiation and arrest of various undifferentiated cancer cell lines thereby preventing unwanted cellular growth and multiplication cycles.

ROS activity in the body can be fairly measured through serum lipid peroxidation product levels. In an observational study done amongst 24 colorectal patient and 24 healthy volunteers, it was found that decreased levels of plasma vitamin $E$ was associated with increased levels of serum lipid peroxidation products (Bhagat et al., 2011). This finding was more pronounced in cancer patients than in the healthy volunteers. Protection from ROS can be the central mechanism for the anticancer potentials of vitamin E. A study done among Australian men showed that tocotirenols (vitamin E with enol groups) were as effective as tocopherols when tested for a number of anti-cancer functions (Ling et al., 2012). Tocotrienols administration showed decreasing levels of serum lipid peroxidases, and thereby lowered oxidative damage. Tocotrienol supplementation also showed the upregulation of pro-apoptotic genes like $\mathrm{BAX}$ and $\mathrm{CD} 40 \mathrm{~L}$ (Ling et al., 2012). A number of other cancer protective responses of vitamin $\mathrm{E}$ like inhibition of epithelial to mesenchymal transformations and down regulation of vascular endothelial growth factors and tumor angiogenic factors and decreased stem cell proliferating factors were also found in this study (Ling et al., 2012). In an observational study done amongst 33 patients with oral cancers due to tobacco smoking in Nigeria, it was observed that serum vitamin E levels were significantly lower in patients having oral cancers compared to the general population (Lawal et al., 2012). The study also found that low serum levels of vitamin $E$ increased the predicted risk for oral caners by 5.6 times (Lawal et al., 2012). In a study done among 103 cervical cancer patients in Mexico, it was observed that patients with increasing disease severity had worsening levels of vitamin E and other anti-oxidant sufficiency scores (Fuchs-Tarlovsky et al., 2010). The vitamin E supplemented groups also showed significant reduction in oxidative stress variables like malondialdehyde (MDA), free carbonyls, dityrosines, and carbonyl/protein rate when compared to the nonsupplemented groups (Fuchs-Tarlovsky et al., 2010). These findings justify the need for inclusion of vitamin $\mathrm{E}$ in SELECT study.

\section{Rationale for Vitamin E Sufficiency Indicators}

The SELECT trial uses serum $\alpha$-tocopherol levels for estimating vitamin E sufficiency (Klein et al., 2000). Behrens et al. (Behrens et al., 1982) had demonstrated that $90 \%$ of the functional vitamin $\mathrm{E}$ in the body is in $\alpha$-tocopherol form and serum levels adequately reflect nutritional status. Horwitt (1960) further demonstrated that there was a linear increase in serum $\alpha$-tocopherol levels with supplementation of any form of vitamin E. The SELECT study administered Vitamin E (dl- $\alpha$ tocopheryl acetate) in doses of $400 \mathrm{IU} /$ day which is less than half the tolerable upper intake of $1000 \mathrm{IU} /$ day. This was well beyond the recommended levels of 22.4-28.4 IU/day (NIH, 2013). In a study done by Farrel and Bieri (Farrell and Bieri, 1975), very high doses of 100 to 800 IU/day did not produce any visible adverse outcomes in a cohort of 28 participants. A number of trials designed to study the effects of vitamin E on cardiovascular and neurodegnerative disorders used supplementations as high as $660 \mathrm{IU} /$ day (Morris et al., 1998; Wen et al., 1999). The preliminaries of the SELECT study used this rationale while deciding about the supplementation strategies. Analysis of these preliminaries also showed encouraging results. Though these results cannot justify such high levels of supplementation in SELECT study, it also cannot be criticized because investigators did not have evidences against the effects of such high levels of supplementation.

Many researchers argue that other markers of vitamin E sufficiency are better than serum $\alpha$-tocopherol. For example, serum $\alpha$-tocopherol/cholesterol ratio takes into consideration the serum lipid levels and thereby more accurately measures vitamin E status (Ford and Sowell, 1999). Many studies have documented an inverse association between serum vitamin E levels and serum cholesterol levels. For example, in a secondary analysis done on NHANES III data, Ford and Sowell (1999) has shown that serum cholesterol significantly and negatively predicted serum vitamin E levels. However, serum 
Table 1. Selected Studies of Selenium, Vitamin E and Prostate Cancer

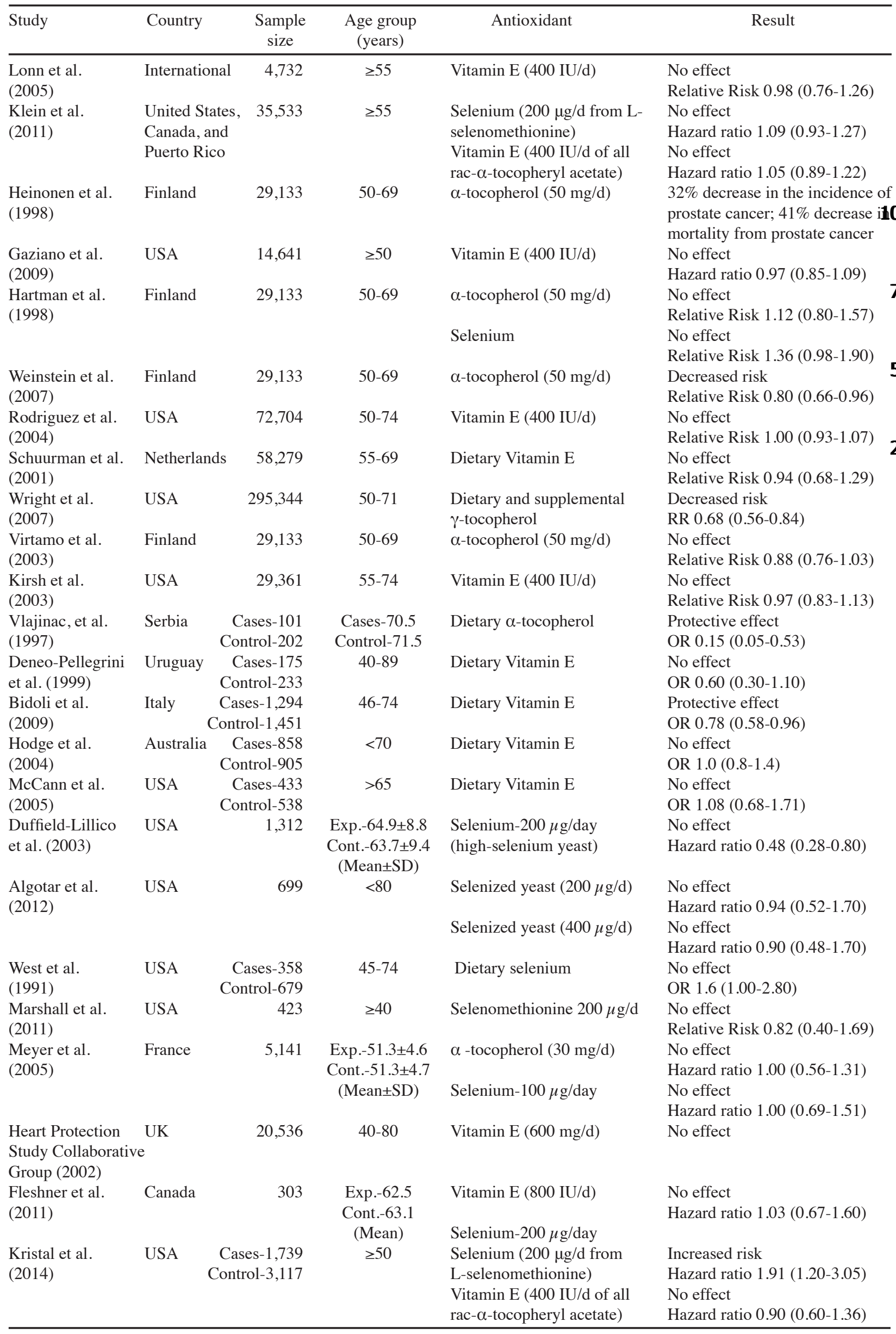


cholesterol levels vary between different age groups, ethnicities, dietary and life style factors and other health related conditions. These differences in cholesterol levels would have compromised the internal validity of the study. Another study done by Vatassery et al. (Vatassery et al., 1983) recommends platelet $\alpha$-tocopherol levels to be more predictive than serum $\alpha$-tocopherols or serum $\alpha$-tocopherol/cholesterol ratio because it did not fluctuate with changing plasma lipid levels. Nevertheless, platelet $\alpha$-tocopherol levels do not reflect fluctuations with skipped supplementations and estimation of compliance would be difficult with this measure.

Table 1 shows selected studies of selenium, vitamin E and prostate cancer (West et al., 1991; Vlajinac et al., 1997; Hartman et al., 1998; Heinonen et al., 1998; Deneo-Pellegrini et al., 1999; Group, 2002; Schuurman et al., 2002; Duffield-Lillico et al., 2003; Virtamo et al., 2003; Hodge et al., 2004; Rodriguez et al., 2004; Lonn et al., 2005; McCann et al., 2005; Meyer et al., 2005; Kirsh et al., 2006; Weinstein et al., 2007; Wright et al., 2007; Bidoli et al., 2009; Gaziano et al., 2009; Fleshner et al., 2011; Klein et al., 2011; Marshall et al., 2011; Algotar et al., 2013; Kristal et al., 2014).

\section{Conclusion}

The SELECT study failed to show any significant risk reduction for prostate cancers ascribable to selenium and vitamin E supplementations. Since the results were contrary to the rationales used for the study, the methodologies used in SELECT study have been argued by many researchers. However, based on the evidences from many epidemiological studies, clinical trials, and pre-clinical studies reviewed here, we can corroborate that SELECT study is a valuable clinical trial with sound methodology. In lieu of the contrary results of the select study, researchers should focus on the probable mechanisms behind these findings and continue their search for newer and effective agents for prevention of prostate cancer.

\section{Acknowledgements}

Authors would like to thank Dr. Marinna Baum and Dr. Adriana Campa for their valuable comments and suggestions. Publication of this article was funded in part by Florida International University Open Access Publishing Fund.

\section{References}

Afanas'ev I (2011). Reactive oxygen species signaling in cancer: comparison with aging. Aging Disease, 2, 219.

Algotar AM, Stratton MS, Ahmann F, et al (2013). Phase 3 clinical trial investigating the effect of selenium supplementation in men at high-risk for prostate cancer. Prostate, 73, 328-35.

Allan CB, Lacourciere GM, Stadtman TC (1999). Responsiveness of selenoproteins to dietary selenium 1,2. Ann Review Nutrit, 19, 1-16.

Behrens W, Thompson J, Madere R (1982). Distribution of alpha-tocopherol in human plasma lipoproteins. Am J Clin Nutrit, 35, 691-6.
Bhagat SS, Ghone RA, Suryakar AN, et al (2011). Lipid peroxidation and antioxidant vitamin status in colorectal cancer patients. Indian J Physiol Pharmacol, 55, 72-6.

Bidoli E, Talamini R, Zucchetto A, et al (2009). Dietary vitamins $\mathrm{E}$ and $\mathrm{C}$ and prostate cancer risk. Acta Oncologica, 48, 890-4.

Brawley OW (2012). Prostate cancer epidemiology in the United States. World J Urol, 30, 195-200.

Brown K, Arthur J (2001). Selenium, selenoproteins and human health: a review. Public Health Nutrit, 4, 593-9.

Burton GW (1994). Vitamin E: molecular and biological function. Proceedings Nutrit Soc, 53, 251-62.

Catalona WJ, Smith DS, Ratliff TL, et al (1991). Measurement of prostate-specific antigen in serum as a screening test for prostate cancer. New Engl J Med, 324, 1156-61.

CDC. 2014. Cancer Prevention and Control [Online]. Available: http://www.cdc.gov/cancer/dcpc/data/men.htm [Accessed October 12 2014].

Cheng W-H, Wu RT, Wu M, et al (2012). Targeting werner syndrome protein sensitizes U-2 OS osteosarcoma cells to selenium-induced DNA damage response and necrotic death. Biochem Biophys Res Comm, 420, 24-8.

Deneo-Pellegrini H, De Stefani E, Ronco A, et al (1999). Foods, nutrients and prostate cancer: a case-control study in Uruguay. Br J Cancer, 80, 591.

Dodig S, Cepelak I (2004). The facts and controversies about selenium. Acta Pharm, 54, 261-76.

Duffield-Lillico A, Dalkin B, Reid M, et al (2003). Selenium supplementation, baseline plasma selenium status and incidence of prostate cancer: an analysis of the complete treatment period of the nutritional prevention of cancer trial. BJU International, 91, 608-12.

Farrell PM, Bieri JG (1975). Megavitamin E supplementation in man. Am J Clin Nutr, 28, 1381-6.

Fleshner NE, Kapusta L, Donnelly B, et al (2011). Progression from high-grade prostatic intraepithelial neoplasia to cancer: a randomized trial of combination vitamin-E, soy, and selenium. J Clin Oncol, 29, 2386-90.

Ford ES, Sowell A (1999). Serun $\alpha$-Tecopherol status in the United States population: findings from the third national health and nutrition examination survey. Am J Epidemiol, 150, 290-300.

Fuchs-Tarlovsky V, Bejarano-Rosales M, Gutierrez-Salmean $\mathrm{G}$, et al (2010). [Effect of antioxidant supplementation over oxidative stress and quality of life in cervical cancer]. Nutricion Hospitalaria, 26, 819-26.

Gaziano JM, Glynn RJ, Christen WG, et al (2009). Vitamins E and $\mathrm{C}$ in the prevention of prostate and total cancer in men: the physicians' health study II randomized controlled trial. JAMA, 301, 52-62.

Group HPSC (2002). MRC/BHF heart protection study of antioxidant vitamin supplementation in 20,536 high-risk individuals: a randomised placebo-controlled trial. Lancet, 360, 23.

Gupta-Elera G, Garrett AR, Robison RA, et al (2012). The role of oxidative stress in prostate cancer. Eur J Cancer Prev, 21, 155-62.

Hartman TJ, Albanes D, Pietinen P, et al (1998). The association between baseline vitamin $\mathrm{E}$, selenium, and prostate cancer in the alpha-tocopherol, beta-carotene cancer prevention study. Cancer Epidemiol Biomarkers Prev, 7, 335-40.

He K (2011). Trace elements in nails as biomarkers in clinical research. European J Clin Investigat, 41, 98-102.

Heinonen OP, Koss L, Albanes D, et al (1998). Prostate cancer and supplementation with $\alpha$-tocopherol and $\beta$-carotene: incidence and mortality in a controlled trial. J Natl Cancer Inst, 90, 440-6.

Ho E, Beaver LM, Williams DE, et al (2011). Dietary factors 
and epigenetic regulation for prostate cancer prevention. advances in nutrition: Intern Rev J, 2, 497-510.

Hodge AM, English DR, McCredie MR, et al (2004). Foods, nutrients and prostate cancer. Cancer Causes Control, 15, 11-20.

Horwitt M (1960). Vitamin E and lipid metabolism in man. Am $J$ Clin Nutrit, 8, 451-61.

Ide H, Lu Y, Yu J, et al (2012). Testosterone promotes DNA damage response under oxidative stress in prostate cancer cell lines. Prostate, 72, 1407-11.

Kheirandish P, Chinegwundoh F (2011). Ethnic differences in prostate cancer. Br J Cancer, 105, 481-5.

Kim J-E, Han M, Hanl K-S, et al (2011). Vitamin E inhibition on platelet procoagulant activity: Involvement of aminophospholipid translocase activity. Thrombosis Res, 127, 435-42.

Kirsh VA, Hayes RB, Mayne ST, et al (2006). Supplemental and dietary vitamin $\mathrm{E}, \beta$-carotene, and vitamin $\mathrm{C}$ intakes and prostate cancer risk. J Natl Cancer Inst, 98, 245-54.

Klein E, Thompson I, Lippman S, et al (2000). SELECT: the selenium and Vitamin E Cancer prevention trial: rationale and design. Prostate Cancer Prostatic Diseases, 3, 145-51.

Klein EA, Thompson IM, Tangen CM, et al (2011). Vitamin E and the risk of prostate cancer: the selenium and Vitamin $\mathrm{E}$ Cancer Prevention Trial (SELECT). JAMA, 306, 1549-56.

Kristal AR, Darke AK, Morris JS, et al (2014). Baseline selenium status and effects of selenium and vitamin E supplementation on prostate cancer risk. J Natl Cancer Inst, 106, djt456.

Laranjinha J, Vieira Ol, Madeira Vt, et al (1995). Two related phenolic antioxidants with opposite effects on vitamin $\mathrm{E}$ content in low density lipoproteins oxidized by ferrylmyoglobin: consumption vs regeneration. Arch Biochem Biophys, 323, 373-81.

Lawal A, Kolude B,Adeyemi B, et al (2012). Serum antioxidant vitamins and the risk of oral cancer in patients seen at a tertiary institution in Nigeria. Nigerian J Clin Practice, 15.

Lemire M, Philibert A, Fillion M, et al (2012). No evidence of selenosis from a selenium-rich diet in the Brazilian Amazon. Environment Int, 40, 128-36.

Levander OA (1997). Selenium requirements as discussed in the 1996 joint FAO/IAEA/WHO expert consultation on trace elements in human nutrition. Biomed Environment Sci, 10, 214-9.

Ling MT, Luk SU, Al-Ejeh F, et al (2012). Tocotrienol as a potential anticancer agent. Carcinogenesis, 33, 233-9.

Liu J-G, Zhao H-J, Liu Y-J, et al (2012). Effect of two selenium sources on hepatocarcinogenesis and several angiogenic cytokines in diethylnitrosamine-induced hepatocarcinoma rats. J Trace Elements Med Biology, 26, 255-61.

Lonn E, Bosch J, Yusuf S, et al (2005). Effects of long-term vitamin $\mathrm{E}$ supplementation on cardiovascular events and cancer: a randomized controlled trial. JAMA, 293, 1338-47.

Marshall JR, Tangen CM, Sakr WA, et al (2011). Phase III trial of selenium to prevent prostate cancer in men with high-grade prostatic intraepithelial neoplasia: SWOG S9917. Cancer Prev Res, 4, 1761-9.

McCann SE, Ambrosone CB, Moysich KB, et al (2005). Intakes of selected nutrients, foods, and phytochemicals and prostate cancer risk in western New York. Nutrit Cancer, 53, 33-41.

Meyer F, Galan P, Douville P, et al (2005). Antioxidant vitamin and mineral supplementation and prostate cancer prevention in the SU. VI. MAX trial. Int J Cancer, 116, 182-6.

Miller AB (2012). New data on prostate-cancer mortality after PSA screening. N Engl J Med, 366, 1047-8.

Morris MC, Beckett LA, Scherr PA, et al (1998). Vitamin E and vitamin $C$ supplement use and risk of incident Alzheimer disease. Alzheimer Dis Assoc Dis, 12, 121-6.
Muir C, Nectoux J, Staszewski J (1991). The epidemiology of prostatic cancer: geographical distribution and time-trends. Acta Oncol, 30, 133-40.

Neve J (1991). Methods in determination of selenium states. $J$ Trace Elements Electrolytes Health Disease, 5, 1-17.

NIH. 2013. Vitamin E fact sheet for health professionals [Online]. Available: http://ods.od.nih.gov/factsheets/ VitaminE-HealthProfessional/ [Accessed September 14 2014].

Powell IJ, Banerjee M, Sakr W, et al (1999). Should AfricanAmerican men be tested for prostate carcinoma at an earlier age than white men? Cancer, 85, 472-7.

Redman C, Scott JA, Baines AT, et al (1998). Inhibitory effect of selenomethionine on the growth of three selected human tumor cell lines. Cancer Letters, 125, 103-10.

Ripple MO, Hagopian K, Oberley TD, et al (1999). Androgeninduced oxidative stress in human LNCaP prostate cancer cells is associated with multiple mitochondrial modifications. Antioxidants Redox Signal, 1, 71-81.

Rodriguez C, Jacobs EJ, Mondul AM, et al (2004). Vitamin E supplements and risk of prostate cancer in US men. Cancer Epidemiology Biomarkers Prev, 13, 378-82.

Roemeling S, Roobol MJ, Kattan MW, et al (2007). Nomogram use for the prediction of indolent prostate cancer. Cancer, 110, 2218-21.

Sakr W, Grignon D, Haas G (1998). Pathology of premalignant lesions and carcinoma of the prostate in African-American men. Sem Urol Oncol, 16, 214-20.

Schröder FH, Hugosson J, Roobol MJ, et al (2009). Screening and prostate-cancer mortality in a randomized European study. New Engl J Med, 360, 1320-8.

Schuurman AG, Goldbohm RA, Brants HA, et al (2002). A prospective cohort study on intake of retinol, vitamins $\mathrm{C}$ and $\mathrm{E}$, and carotenoids and prostate cancer risk (Netherlands). Cancer Causes Control, 13, 573-82.

Shimizu H, Ross R, Bernstein L, et al (1991). Cancers of the prostate and breast among Japanese and white immigrants in Los Angeles County. Br J Cancer, 63, 963-6.

Steyerberg E, Roobol M, Kattan M, et al (2007). Prediction of indolent prostate cancer: validation and updating of a prognostic nomogram. J Urology, 177, 107-12.

Swanson C, Patterson B, Levander O, et al (1991). Human [74Se] selenomethionine metabolism: a kinetic model. Am J Clin Nutrit, 54, 917-26.

Thompson Jr IM, Goodman PJ, Tangen CM, et al (2013). Longterm survival of participants in the prostate cancer prevention trial. New Engl J Med, 369, 603-10.

Torricelli P,Caraglia M,Abbruzzese A, et al (2013). $\gamma$-Tocopherol inhibits human prostate cancer cell proliferation by upregulation of transglutaminase 2 and down-regulation of cyclins. Amino Acids, 44, 45-51.

Vatassery G, Krezowski A, Eckfeldt J (1983). Vitamin E concentrations in human blood plasma and platelets. Am J Clin Nutrition, 37, 1020-4.

Venkateswaran V, Klotz LH (2010). Diet and prostate cancer: mechanisms of action and implications for chemoprevention. Nature Reviews Urology, 7, 442-53.

Virtamo J, Pietinen P, Huttunen J, et al (2003). Incidence of cancer and mortality following alpha-tocopherol and betacarotene supplementation: a postintervention follow-up. JAMA, 290, 476-85.

Vlajinac H, Marinkovic J, Ilic M, et al (1997). Diet and prostate cancer: a case-control study. Eur J Cancer, 33, 101-7.

Vykhovanets EV, Shankar E, Vykhovanets OV, et al (2011). High-fat diet increases NF- $x \mathrm{~B}$ signaling in the prostate of reporter mice. Prostate, 71, 147-56.

Weinstein SJ, Wright ME, Lawson KA, et al (2007). Serum and 
dietary vitamin $\mathrm{E}$ in relation to prostate cancer risk. Cancer Epidemiology Biomarkers Prev, 16, 1253-9.

Wen Y, Killalea S, Norris L, et al (1999). Vitamin E supplementation in hyperlipidaemic patients: effect of increasing doses on in vitro and in vivo low-density lipoprotein oxidation. Euro J Clin Investigation, 29, 1027-34.

West DW, Slattery ML, Robison LM, et al (1991). Adult dietary intake and prostate cancer risk in Utah: a case-control study with special emphasis on aggressive tumors. Cancer Causes Control, 2, 85-94.

WHO (2008). World cancer factsheet [Online]. Available: http://gicr.iarc.fr/files/resources/20120906WorldCancerFactSheet.pdf [Accessed September 72014 ].

Wright ME, Weinstein SJ, Lawson KA, et al (2007). Supplemental and dietary vitamin $\mathrm{E}$ intakes and risk of prostate cancer in a large prospective study. Cancer Epidemiol Biomarkers Prev, 16, 1128-35.

Wu M, Kang MM, Schoene NW, et al (2010). Selenium compounds activate early barriers of tumorigenesis. $J$ Biological Chemistry, 285, 12055-62.

Wu M, Wu RT, Wang TT, et al (2011). Role for p53 in seleniuminduced senescence. J Agricultural Food Chemistry, 59, 11882-7.

Xia S-J, Cui D, Jiang Q (2012). An overview of prostate diseases and their characteristics specific to Asian men. Asian $J$ Andrology, 14, 458.

Xia Y, Hill KE, Burk RF (1989). Biochemical studies of a selenium-deficient population in China: measurement of selenium, glutathione peroxidase and other oxidant defense indices in blood. J Nutrit, 119, 1318-26.

Yang L, Pascal M, Wu XH (2013). Review of selenium and prostate cancer prevention. Asian Pac J Cancer Prev, 14, 2181-4.

Yoshizawa K, Willett WC, Morris SJ, et al (1998). Study of prediagnostic selenium level in toenails and the risk of advanced prostate cancer. J Natl Cancer Inst, 90, 1219-24. 